\title{
Perturbation of operator algebras
}

by

Erik Christensen

\section{Introduction.}

The Banach-Stone theorem says in the language we will use, that if two commutative $\mathrm{C}^{*}$-algebras are isometrical isomorphic, then they are isomorphic as $C^{*}$-algebras. In this formulation there are two natural questions, does the theorem hold for non commutative $C^{*}$-algebras, and is it possible to replace isometrical isomorphic with nearly isometrical isomorphic.

A complete answer to the first question was given by Kadison in [9], and M. Cambern settled the second in the afirmative in [5].

In section 3 we show that Cambern's result may be partially extended to non commutative von Neumann algebras having the property that any normal representation of the algebra has property $P([17],[18])$. We call this property $M$, (M for mixing [1]]).

In section 2 we show that the class of von Neurnann algebras having property $M$ is closed under various operations, and that it contains type $I$ and hyperfinite algebras.

When we have the result of section 3 that a nearly isometric completely positive map of a von Neumann algebra having property $M$ is close to a * isomorphism between the algebras, we nearly have proved that von Neumann algebras whose unitballs are close in the Hausdorff metric, are unitatirly equivalent via a unitary close to the identity.

The only thing we then have to prove is, that if $\Phi$ is an isomorphism of a von Neumann algebre A having property $M$ onto a von Neumann algebra $B$, acting on the same Hilbertspace, and $\Phi$ satisfies for some positive $k$ less than one and any $x$ in $A$ 


$$
\|x-\Phi(x)\| \leq k\|x\|
$$

then $\Phi$ is implemented by a unitary in $(A \cup B) "$, whose distance to the identity is less than $2^{\frac{1}{2}} \mathrm{k}$. (Proposition 4.2).

In the end of section 4 we show how this proposition, which holds for any von Neumann algebra if $B=A$, is the keypoint in the sence that we may replace property $M$, in the theorems where we use it, by the property that proposition 4.2 is valid for any von Neumann algebra isomorphic to A.

Section 5 is devoted to the study of perturbations of $C^{*}$-algebras, and we show that the general hypothesis that close algebras are isomorphic via a unitary close to the identity, is true for the most elementary algebras, namely commutative $\mathrm{C}^{*}$ algebras and ideal $C^{*}$-algebras.

Finally we show that neighbouring representations sometimes are unitarily equivalent.

I want to thank professor E. St $\not$ rmer for his help and kind hospitality during my stay at the University of Oslo. I also want to thank G. Elliot for drawing my attention to the work [5] of M. Cambern.

\section{Notation and preliminaries.}

A von Noumann algebra acting on a Hilbertspace $H$ is a weakly closed selfadjoint subalgebra of the algebra $B(H)$ of all abounded operators on $H$, which contains the identity $I$ of $B(H)$. In the former papers, [G], [12], [14] on perturbation theory, the identity of $B(H)$ is not assumed to be in the algebras under consideration, but this restriction makes computations simpler, and it is of nothing but technical interest for the theory, not to restrict the problems to this case.

Let $A$ be a von Neumann algebra acting on a Hilbertspace $H$, then we will

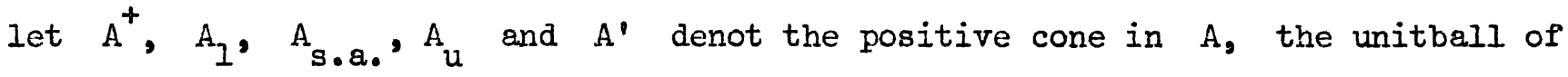


$A$, the selfadjoint part of $A$, the group of unitaries in $A$ and the commutant of $A$.

For an element $x$ in $B(H)$ we write $\overline{c o}_{A}(x)$ for the weak closure of the convex hull of the set of all operators $u^{*} x u$, with $u$ a unitary in $A$.

We say that $x$ is mobile relative to $A$ if $\overline{c o}_{A}(x) \cap A^{\prime} \neq \emptyset$ and that $A$ is mixing or has property $P$ if every $x$ in $B(H)$ is mobile relative to $A$ $([1],[18])$.

Unfortunately we do not know if property $P$ is an algebraic invariant for a von Neumann algebra, so we have to introduce the following.

\subsection{Definition.}

A von Neumann algebra $A$ has property $M$ if any von Neumann algebra $B$, isomorphic to $A$ has property $P$.

\subsection{Proposition.}

Let $A$ be a von Neumann algebra on a Hilbertspace $H$.

(i) If $A$ is generated by a family $\left(A_{\alpha}\right)_{\alpha \in D}$ of mutually commuting sub von Neumann algebras having property $M$, Then A has property $M$.

(ii) If $A$ is generated by an increasing directed set $\left(A_{\alpha}\right)_{\alpha \in D}$ of sub von Neumann algebras having property $M$, then $A$ has property $M$. Proof.

The proof in [17], 4.4.16 proposition p.p. 208-209 applies to property M as well as to property $P$.

\subsection{Remark.}

Any type I von Neunann algebra and any hyperfinite algebra has property $M$ ([17], 4.4.17), any finite or infinite tensorproduct of algebras having property $M$, has property $M\left(2.2\left(i^{i}\right)\right)$.

Suppose that $A$ is a von Neumann algebra on a Hilbertspace $H$ and that $A$ 
contrins a weakly dense sub $C^{*}$-algebra $\widetilde{C}$ which is strongly amenable ([4] Def. 2 p.564), we can without loss of generality assume that I belongs to $O C$. The predual of $B(H)$ is a Banach or module with the canonical products $(a, \varphi) \rightarrow \varphi\left(a_{0}\right), \quad(\varphi, a) \rightarrow \varphi(. a)$. Let $x$ be in $B(H)$ then the map $a \rightarrow[a, x]$ is a derivation of $O L$ into $B(H)$, so there exists

$z \in \overline{c o}\left\{(u x-x u) u^{*} \mid u \in O u_{u}\right\}=\overline{c o}_{o}(x)-x$, such that $[a, x]=-[a, z]$ for all $a$ in 0 . Write $z=y-x$ then for eech $a$ in $\pi[a, x]=-([a, y]-[a, x])$, hence $y \in A^{\prime}$ and A has property $M$.

2.4 Definition.

For subspaces $R$ and $S$ of a mormed space

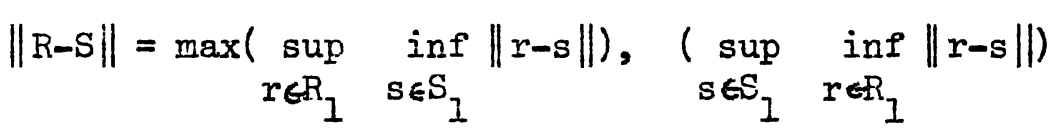

\subsection{Theorem}

Let $A$ and $B$ be von Neumann algebras on a Hilbertspace $H$. If $\|A-B\| \leq k \leq \frac{1}{2}$ and $A$ and $B$ both have property $M$ then $\left\|A^{\prime}-B^{\prime}\right\| \leq 2 k$.

Proof.

Let $x \in\left(A^{\prime}\right)_{I}$ take $y \in{\overline{\mathrm{CO}_{B}}}_{B}(x) \cap B^{\prime}$ then

$$
\|x-y\| \leq \sup _{u \in B_{u}}\left\|x-u^{*} x u\right\|=\sup _{u \in B_{u}}\|u x-x u\|
$$

for each $u$ in $B_{u}$ there exists $z$ in $A$ such that

$$
\|z-u\| \leq k, \text { then }\|u x-x u\|=\|(u-z) x-x(u-z \| \leq 2 k \text { and }\|x-y\| \leq 2 k \text {. }
$$

The theorem follows.

We think that property $M$ is redundent in Theorem 2.5 and an indication is seen in the next proposition. 


\begin{abstract}
2.6 Proposition.
Let $A$ and $B$ be von Neumann algebras on a Hilbertspace $H$ if $\|A-B\| \leq k<\frac{1}{2} \quad$ then $\quad B^{\prime} \subseteq\left(A^{\prime} \cup B\right)^{\prime \prime}$.
\end{abstract}

Proof.

Let $p$ be a projection in $A \cap B^{\prime}$ then there exists a projection $q$ in $B$ such that $\|p-q\| \leq 2 k$. ([6] Lemma 2.1). Since $p$ and $q$ commute and $2 k<1$ we must have $p=q$ so $A \cap B^{\prime} \subseteq B$ which is equivalent to the statement in the proposition.

It seems surprising that $\|A-B\|<\frac{1}{2}$ implies $A \cap B^{\prime}$ is abelian.

\title{
3. Nearly isometric mappings.
}

In [9] Kadison proved a non comutative version of the Banach-Stone theorem, and showed that a positive isometry between $C^{*}$ algebras is a Jordanhomomorphism. Another point of view is found in [5] where M. Cambern shows that commutative $c^{*}$ algebras $\sigma$ and $B$, which are nearly isomorphic as Banach-spaces are isomorphic as $C^{*}$ algebras.

What follows here is that to a completely positive nearly isometric map $\Phi$ of a von Neumann algebra $A$ having property $M$ onto another $B$, there exists a * isomorphism $\psi$ of $A$ onto $B$ such that $\|\Phi-\psi\|$ is small.

Let $n$ be a natural number and let $M_{n}$ be the algebra of all complex $\mathrm{n} \times \mathrm{n}$ matrices, a linear map $\Phi$ of a $\mathrm{C}^{*}$ algebra of into $\mathrm{B}(\mathrm{H})$ for some Hilbertspace $H$ is completely positive, if the map $\Phi_{n}$ of $\sigma \otimes M_{n}$ into $B(H) \otimes M_{n}$ given by $\Phi_{n}\left(a_{i j}\right)=\left(\Phi\left(a_{i j}\right)\right)$ is positive for each n. In [l] Arveson discusses completely positive maps and gives a proof of Stinesprings theorem [19] which we want to formulate in the following way.

\subsection{Theorem (Stinespring).}

Let $a$ be a $C^{*}$ algebra with identity, let II be a Hilbertspace and let $\Phi$ be a completely positive linear map of $O$ into $B(H)$ satisfying $\Phi(I)=I$, then 
there exists a Hilbertspace $K$ containing $H$ and a representation II of $O C$ into $B(K)$ such that for the projection $p$ of $K$ onto $H$ and $x$ in $O$.

$$
\Phi(x)=p \Pi(x) \mid H
$$

3.2 Remerk.

That if $\mathcal{O}$ is a von Neumann algebra and $\Phi$ is normal, then it is easily checked that $I$ can be chosen normal.

\subsection{Lemma.}

Let $\Phi$ be a normal completely positive map of a von Neumann algebra A having property $M$ into a von Neumann algebra $B$ on a Hilbertspace $H$, which satisfies $\Phi\left(I_{A}\right)=I_{B}$.

Let $t$ be a positive real number $0<t<\frac{1}{4}$, if for each unitary $u$ in $A$

$$
\Phi\left(u^{*}\right) \Phi(u) \geq(1-t) I
$$

then there exists a normal homomorphism $\psi$ of $A$ into $B$, with

$$
\|\Phi-\psi\| \leq 4(2 t)^{\frac{1}{2}}\left(1+(1-4 t)^{\frac{1}{2}}\right)^{-\frac{1}{2}}
$$

Proof.

We use the notation in 3.1 and 3.2 so $I$ is a non degenerated normal representation of $A$ into $B(K)$, hence $\Pi(A)$ is a von Neumann algebra ([7] ch.I $\$ 4.3 \operatorname{Cor} .2$ p.54).

Let $E$ be the central support projection for $\Pi$ then $(I-E) A(I-E) \oplus \Pi(A)$ is a von Neumann algebra isomorphic to $A$, hence it has property $P$, so it follows that $\Pi(A)$ has property $P$. Choose $r$ in $\overline{c o}_{\Pi(A)}(p) \cap \Pi(A)$ ' where $p$ is the projection onto $H$ then 


$$
\begin{aligned}
& \|r-p\| \leq \sup _{u \in A}\|p \Pi(u)-\Pi(u) p\|= \\
& \sup \|p I I(u)(I-p)-(I-p) \Pi(u) p\|= \\
& u \in A_{u} \\
& \sup _{u \in A}\left\|(I-p) \Pi\left(u^{*}\right) p \Pi(u)(I-p)+p \Pi\left(u^{*}\right)(I-p) \Pi(u) p\right\|^{\frac{1}{2}}= \\
& \sup _{u \in \Lambda}\left(\operatorname { m e x } \left(\left\|p \Pi\left(u^{*}\right)(I-p) \Pi(u) p\right\|^{\frac{1}{2}}\right.\right. \text {, } \\
& \left.\left.\left\|\operatorname{pI}(u)(I-p) \Pi\left(u^{*}\right) p\right\|^{\frac{1}{2}}\right)\right)= \\
& \sup _{u \in A}\left\|p \Pi\left(u^{*}\right) \Pi(u) p-p \Pi\left(u^{*}\right) p \Pi(u) p\right\|^{\frac{1}{2}}= \\
& \sup _{u \in A}\left\|I-\Phi\left(u^{*}\right) \Phi(u)\right\|^{\frac{1}{2}} \leq t^{\frac{1}{2}}
\end{aligned}
$$

The argument in the proof of Lemma 2.1 of [6] shows that the spectral projection $q$ for $r$ corresponding to the interval $\left[t^{\frac{1}{2}}, 1\right]$ has distance less than $2 t^{\frac{1}{2}}$ from $p$, so for the operator $x=q p+(I-q)(I-p)$ we obtain

$$
\|I-x\|=\|(2 q-I)(q-p)\|=\|q-p\| \leq 2 t^{\frac{1}{2}}<1
$$

Let $x=u z$ be the polardecomposition of $x$ then Lemma 2.7 in [6] shows that $u$ is unitary and

$$
\|I-u\| \leq 2^{\frac{1}{2}}\left(2 t^{\frac{1}{2}}\right)\left(1+(I-4 t)^{\frac{1}{2}}\right)^{-\frac{1}{2}}
$$

Since $B$ is a von Neumann algebra and $(\Pi(A) \cup p)^{\prime \prime}$ is the veak closure of of the algebra generated by $\Pi(A)$ and $p$, we have $p(\pi(A) \cup p) " p$ is contained in $B$, moreover since $q$ is in $\Pi(A) \cdot n(\pi(A) \cup p)^{\prime \prime}, u$ is in $(\pi(A) \cup p)^{\prime \prime}$ and satisfies $u^{*} u^{*}=q$, so we may construct a homomorphism $\psi$ of $A$ into $B$ by $\forall a \in A: \psi(a)=p u^{*} \Pi(a) u p$ 
For each a in $A_{1}$

$$
\begin{aligned}
& \|\psi(a)-\Phi(a)\|=\left\|p u^{*} \Pi(a) u p-p \Pi(a) p\right\|=\|q(\Pi(a) u-u \Pi(a)) p\| \leq \\
& \|\Pi(a) u-u \Pi(a)\| \leq 2\|I-u\| \leq 4(2 t)^{\frac{1}{2}}\left(1+(1-4 t)^{\frac{1}{2}}\right)^{-\frac{1}{2}} .
\end{aligned}
$$

\subsection{Theorem.}

Let $\Phi$ be a completely positive map of a von Neumann algebra A having property $M$ onto a von Neumann algebra $B$ and let $t$ be a positive real number in the interval $[0,1 / 84]$.

If $\Phi$ satisfies $\|\Phi\| \leq 1$ and $\left\|\Phi^{-1}\right\| \leq(1+t)$ then there exists an isomorphism $\psi$ of A onto B such that

$$
\|\Phi-\psi\| \leq 8,5 t^{\frac{1}{2}}+7 t
$$

Proof.

The proof is divided into some lemmas, some of which we will use later.

\subsection{Lerama.}

Let $\Phi$ be a positive map of a von Neumann algebra $A$ into a $C^{*}$ algebra $B$, and suppose that for some real positive $t$ we have

$$
(1+t)^{-1}\|a\| \leq\|\Phi(a)\| \leq \| \text { a } \| \text { for all a in } A
$$

then any $z$ in $A_{I}$ with $\Phi(z) \geq 0$ will satisfy $z \geq-t I$.

\section{Proof.}

Suppose $z$ is in $A_{1}$ and $\Phi(z) \geq 0$, to any non negative real number $s$ which is less than or equal to the norm of the negative part of $z$ there exists a spectral projection $e$ for $z$ such that

$$
\begin{aligned}
& \mathrm{z} \leq-\mathrm{se}+(I-\mathrm{e})=I-(I+s) \mathrm{e} \quad \text { so } \\
& 0 \leq \Phi(\mathrm{z}) \leq \Phi(I)-(I+s) \Phi(e) \leq I-(I+s) \Phi(e) \\
& I=\|I\| \geq(I+s)\|\Phi(e)\| \geq \frac{(I+s)}{(I+t)}\|e\| \text {. The Ierma follows. }
\end{aligned}
$$


Let $A$ be a von Neumann algebra and $\varphi$ a positive linear functional on A, then $\varphi$ is called singular if $\varphi$ dominates no positive normal functionals. The linear span of the positive singular functionals on $A$ is denoted by $A_{*}^{\perp}$, we write $A_{*}$ for the predual of $A$ and $A^{*}$ for the dual, and have $A^{*}=A_{*}^{+} \oplus_{1} A_{*}$, which means that the norm on $A^{*}$ is the sum of the norms on the components.

In ([2I], Th 1) Tomiyama shows, how this splitting of the dual space implies that any uniformly continuous linear mapping $\Phi$ of $A$ into another von Neumann algebra $B$ has a unique decomposition $\Phi=\Phi^{\mathrm{n}}+\Phi^{\mathbf{S}}$ into a $\sigma$-weakly continuous part and a singular part. We just sketch the proof. Let $\Phi^{t} \mid \mathrm{B}$ be the restriction of the transposed of $\Phi$ to $B$, and let $\tilde{\Phi}$ be the transposed of this map.

In $A^{* *}$ there exists a central projection $p$ such that

$$
\left\{x \in A^{* *} \mid f(x)=0, \forall f \in A_{*}\right\}=(1-p) \Lambda^{* *}
$$

then $\Phi^{\mathrm{n}}$ and $\Phi^{\mathrm{S}}$ are defined such that for each a in $\mathrm{A}$

$$
\Phi^{\mathrm{n}}(a)=\tilde{\Phi}(\mathrm{pa}) \text { and } \Phi^{\mathrm{s}}(a)=\tilde{\Phi}((1-p) a) \text {. }
$$

It is easy to verify that $\tilde{\Phi}$ is completely positive whenever $\Phi$ is so, and Theorem 2.1 shows that the map $a \rightarrow$ pa is completely positive. So we get that both $\Phi^{\mathrm{n}}$ and $\Phi^{\mathrm{S}}$ are completely positive/positive if $\Phi$ is so.

\subsection{Leroma.}

Let $\Phi$ be a positive map of a von Neumann algebra A ontc a von Neumann algebra $B$, and $\Phi=\Phi^{n}+\Phi^{S}$ the canonical decomposition of $\Phi$ into it's normal and singular perts. If for a real number $t$ in $[0,1]$,

$$
\|\Phi\| \leq 1 \text { and }\left\|\pi^{-1}\right\| \leq I+t \text {, then }\left\|\Phi^{s}\right\| \leq t
$$


Proof.

Let $S$ be the set of all positive normal functionals on $B$ with norm 1 , fix an $f$ in $S$ and consider the set of families of pairwise orthogonal projections from the kernel of $\rho \circ \Phi^{8}$, use Zorn's lemma to obtain a maximal family $\left(p_{\alpha}\right)_{\alpha} \in D^{*}$ If $r=\sum_{\alpha \in D}$ is different to $I$, then since the functional $f \circ \Phi^{s}$ is positive and singular there exists a projection $q$ in $A$ such that $q \leq I-r, q \neq 0$ and $f \circ \Phi^{S}(q)=0$, contradicting the maiximality of $\left(p_{\alpha}\right)_{\alpha \in} \cdot([20] \mathrm{Th} .3$ Cor.I p.197). Define $z$ in $B$ and $x$ in $A$ by $z=\Phi(I)-\sum_{\alpha \in D} \Phi\left(p_{\alpha}\right)$ and $x=\Phi^{-1}(z)$, then for any finite subset $D_{0}$ of $D, \Phi\left(I-\sum_{\alpha \in D} p_{\alpha}-x\right) \geq 0$ and so lemma 3.5 applies and we obtain $-t I \leq I-\sum_{\alpha \in D_{0}} p_{\alpha}-x$, hence $x \leq t I$ and $0 \leq z=\Phi(x) \leq$ ' $I$ $t \Phi(I) \leq t I$.

For any finite subset $D_{0}$ of $D$ we get

$$
\left\|f \circ \Phi^{S}\right\|=\mid \rho \circ \Phi^{S}\left(I-\sum_{\alpha} \in_{0} p_{\alpha}\right) \leq f \circ \Phi\left(I-\sum_{\alpha \in D_{0}} p_{\alpha}\right)+f(x) \leq\|\dot{x}\| \leq t .
$$

which shows that $\left\|\Phi^{s}\right\| \leq t$.

Proof of Theorem 3.4 .

Choose $z$ in A such that $\Phi(z)=I$, then the Schwarz-inequality in ([13] Theorem 2, p.4) shows that

$$
\begin{aligned}
& \Phi\left(z^{2}\right) \geq \Phi(z) \Phi(I)^{-I} \Phi(z)=\Phi(I)^{-I} \\
& \left\|\Phi(I)^{-I}\right\| \leq\left\|\Phi\left(z^{2}\right)\right\| \leq\left\|z^{2}\right\|=\|z\|^{2} \leq(I+t)^{2}\|\Phi(z)\|^{2}=(I+t)^{2}
\end{aligned}
$$

by using Lemma 3.6 and (1) we obtain

$$
\Phi^{n}(I) \geq\left((I+t)^{-2}-t\right) I>\frac{39}{100} I \text { for } t<1 / 4
$$

We may then normalize $\Phi^{n}$ and get a completely positive nommal map $\Gamma$ of A into $B$ which satisfies

$$
\begin{array}{ll}
\Gamma(I)=I \quad \text { by defining } \\
\Gamma(x)=\Phi^{n}(I)^{-\frac{1}{2}} \Phi^{n}(x) \quad \Phi^{n}(I)^{-\frac{1}{2}} .
\end{array}
$$


Next we want to show that $\Gamma$ maps $A$ onto $B$, and to compute bounds for $\Gamma^{-1}$ and $\|\Phi-\Gamma\|$.

We start with $\|\Phi-\Gamma\|$ let $\mathbf{x}$ be in $A$ if we then use (2) and Lemma 3.6

$$
\|(\Gamma-\Phi)(x)\| \leq\left\|\Gamma(x)-\Phi^{n}(I)^{\frac{1}{2}} \Gamma(x) \Phi^{n}(I)^{\frac{1}{2}}\right\|+\left\|\Phi^{s}(x)\right\| \leq\left(2\left\|I-\Phi^{n}(I)^{\frac{1}{2}}\right\|+t\right)\|x\|
$$

$$
\|\Gamma-\Phi\| \leq 2\left((I+t)-(I+t)^{-2}\right)+t \leq 7 t
$$

Lemma 3.6 is used again to obtain a bound for $\Gamma^{-1}$.

$$
\|x\| \leq(1+t)\left\|\Phi^{n}(x)+\Phi^{s}(x)\right\| \leq(1+t)\|\Gamma(x)\|+t(1+t)\|x\|
$$

$$
\left.\forall x \in A:(1-t-t)^{2}\right)(1+t)^{-1}\|x\| \leq\|r(x)\| \leq\|x\|
$$$$
\forall x \in A:(1+1 / 4)^{-1}\|x\| \leq\|r(x)\| \leq\|x\|
$$

It is now clear that $\Gamma(A)$ is a normclosed subspace and further $\Gamma(A)$ has the property that for any $y$ in $B$ there exists $z_{1}$ in $\Gamma(A)$ such that $\left\|y-z_{1}\right\| \leq 3 / 4\|y\|$, take just $z_{1}$ to be $\Gamma\left(\Phi^{-1}(y)\right)$

$$
\left\|y-z_{1}\right\|=\left\|(\Phi-\Gamma) \Phi^{-1}(y)\right\| \leq 7 t(1+t)\|y\| \leq 3 / 4\|y\| \text {. }
$$

Continue in this way i.e. to $y-z_{1}$ find $z_{2}$ in $\Gamma(\Lambda)$ with $\left\|\left(y-z_{1}\right)-z_{2}\right\| \leq 3 / 4\left\|y-z_{1}\right\| \leq(3 / 4)^{2}\|y\|$

$\left\|\left(y-z_{1} \cdots-z_{n-1}\right)-z_{n}\right\| \leq(3 / 4)^{n}\|y\|$ we may conclude that $\Gamma$ maps $A$ onto $B$.

The next step is to show that $\Gamma$ carries a unitary over into a nearly unitary operator. To this purpose we want to introduce the objects $(\pi, K, p)$ from Theorem 3.I for the completely positive map $\Gamma$.

Pick a unitary $u$ in $A$ and suppose that there exists a projection $q$ in $B$ such that $\Gamma(u) q=0$, then for $x=\Gamma^{-1}(q)$ Lemma 3.5 and (4') yields

$$
-I / 4 I \leq x \leq 5 / 4 I \quad \text { and } \quad\|x\| \geq 1
$$

Consider $\Gamma(u x)$ and $\Gamma(u) \Gamma(x)$, the former has norm greater than or equal to $4 / 5$, 
while the latter is equal to zero, if we just recall that $\Gamma(x)^{2}=\Gamma(x)$, then the following computation is straight forward.

$$
\begin{aligned}
& 4 / 5 \leq\|\Gamma(u x)\|=\|\Gamma(u x)-\Gamma(u) \Gamma(x)\|=\left\|p \Pi(u)\left(I_{K}-p\right) \Pi(x) p\right\| \leq \\
& \left\|p \Pi(x)^{*}\left(I_{K}-p\right) \Pi(x) p\right\|^{\frac{1}{2}}=\left\|\Gamma\left(x^{2}\right)-\Gamma(x)^{2}\right\|^{\frac{1}{2}}= \\
& \left\|\Gamma\left(x^{2}-x\right)\right\|^{\frac{1}{2}} \leq\left\|x^{2}-x\right\|^{\frac{1}{2}} \leq(5 / 16)^{\frac{1}{2}}<4 / 5
\end{aligned}
$$

We continue to look at $\Gamma(u)$, let wa be the polardecomposition of $\Gamma(u)$. Then we have just proved that $w$ is unitary, and now we want to show that a is close to $I$.

It is well known that $\Gamma(u)$ is the average of the two unitaries

$$
\Gamma(u)+i w\left(I-a^{2}\right)^{\frac{1}{2}} \quad ; \quad \Gamma(u)-i w\left(I-a^{2}\right)^{\frac{1}{2}}
$$

Define $x$ to be $\Gamma^{-1}\left(i w\left(I-a^{2}\right)^{\frac{1}{2}}\right)$ then by (4),

$$
\begin{array}{ll}
\|u \pm x\| \leq k=(1+t)\left(1-t-t^{2}\right)^{-1} & \text { so } \\
u^{*} u+x^{*} x \leq k^{2} I & \text { and } \\
x^{*} x \leq\left(k^{2}-I\right) I &
\end{array}
$$

The Schwarz-inequality $\Gamma\left(x^{*}\right) \Gamma(x) \leq \Gamma\left(x^{*} x\right)$ for completely positive maps is then used and we get the following inequality, which enables us to use Lemma 3.1.

$$
\begin{aligned}
& I-\Gamma\left(u^{*}\right) \Gamma(u)=I-a^{2}=\Gamma\left(x^{*}\right) \Gamma(x) \leq \Gamma\left(x^{*} x\right) \leq\left(k^{2}-I\right) I= \\
& \left((I+t)^{2}-\left(1-t-t^{2}\right)^{2}\right)\left(1-t-t^{2}\right)^{2} \leq 4 t+7 t^{2}
\end{aligned}
$$

Let $\psi$ be a homomorphism of $A$ into $B$ which satisfies

$$
\begin{aligned}
& \|\psi-\Gamma\| \leq 4\left(8 t+14 t^{2}\right)^{\frac{1}{2}}\left(1+\left(1-16 t-28 t^{2}\right)^{-\frac{1}{2}} \leq\right. \\
& 4(8,2 t)^{\frac{1}{2}}(11 / 6)^{-\frac{1}{2}}<8,5 t^{\frac{1}{2}}<1 .
\end{aligned}
$$

We may then repeat the argument, which showed that $\Gamma$ is onto, to show that $\psi$ is onto and $\psi$ is an isomorphism of $A$ onto $B$ with $\|\psi-\Phi\| \leq 8,5 t^{\frac{1}{2}}+7 t$. 


\section{Perturbation of von Neumann algebras.}

Recall that a von Neumann algebra A on a Hilbertspace II has extension property if there exists a projection of norm one from $B(H)$ onto A. ([23]).

It is well known that if $A$ has property $P$ then $A^{\prime}$ has extension property (Proposition 4.4.15 [17]), but then A has extension property ([22]) so property $M$ is stronger than extension property.

\subsection{Theorem.}

Two von Neumann algobras $A$ and $B$ on a Hilbertspace $H$ for which $\|A-B\|<1 / 169$ are isomorphic via a unitary $u$ in $(A \cup B) "$ with $\|I-u\| \leq 19\|A-B\|^{+\frac{1}{2}}$, if $A$ has property $M$ and $B$ has extension property.

\section{Proof.}

Let $\pi$ be a projection of norm one frorn $B(H)$ onto $B$ and let $\Phi$ be the restriction of $\Pi$ to $A$, then $\Phi$ is completely positive ([24]; Th. 1 ).

Define $k=\|A-B\|$ and let $x$ be in $A$, then since $I$ is a projection $\|\Pi(x)-x\|=\|\Pi(x-y)-(x-y)\|$ for any $y$ in $B$, so

$$
\|x\| \geq\|\Phi(x)\|=\|\Pi(x)\| \geq\|x\|-\|x-\Pi(x)\| \geq\|x\|-2 k\|x\|=\left(1+2 k(1-2 k)^{-1}\right)^{-1}\|x\| .
$$

If we decompose $\Phi$ into its normal and singular parts $\Phi^{\mathrm{n}}$ and $\Phi^{\mathrm{s}}$ we find as in section 3 that $\Phi^{\mathrm{n}}$ is completely positive and $\left\|\Phi^{5}\right\| \leq 2 \mathrm{k}(1-2 \mathrm{k})^{-1}$.

Since the identities of $A$ and $B$ coincide we get

(I) $\quad\left\|I-\Phi^{n}(I)\right\|=\left\|\Phi^{s}(I)\right\| \leq 2 k(I-2 k)^{-1}<1$.

and we may normalize $\Phi^{\mathrm{n}}$. Call the normalized map $\Gamma$.

(2) $\quad \Gamma(x)=\Phi^{n}(I)^{-\frac{1}{2}} \Phi^{n}(x) \Phi^{n}(I)^{-\frac{1}{2}}$ for all $x$ in $A$.

For any unitary $u$ in $A$ we have the norm of the elemetn $z$ given by $z=(u-\Phi(u))+\Phi^{s}(u)$ is less than or equal to $2 k\left(1+(1-2 k)^{-1}\right)$. 


$$
\begin{aligned}
& \Gamma\left(u^{*}\right) \Gamma(u)=\Phi^{n}(I)^{-\frac{1}{2}} \Phi^{n}\left(u^{*}\right) \Phi^{n}(I)^{-I} \Phi^{n}(u) \Phi^{n}(I)^{-\frac{1}{2}} \geq \\
& \Phi^{n}(I)^{-\frac{1}{2}} \Phi^{n}\left(u^{*}\right) \Phi^{n}(u) \Phi^{n}(I)^{-\frac{1}{2}}= \\
& \Phi^{n}(I)^{-\frac{1}{2}}\left(\left(u^{*}-z^{*}\right)(u-z)\right) \Phi^{n}(I)^{-\frac{1}{2}} \geq \\
& (I-2\|z\|) \Phi^{n}(I)^{-I} \geq\left(1-4 k\left(I+(I-2 k)^{-I}\right)\right) I \geq(1-8,15 k) I
\end{aligned}
$$

According to Lemma 3.3 there exists a homomorphism $\psi$ of A into B with

$$
\|\psi-\Gamma\| \leq 4(16,3 k)^{\frac{1}{2}}\left(1+(1-32,6 k)^{\frac{1}{2}}\right) \leq 12,1 k^{\frac{1}{2}}
$$

For any $\mathrm{x}$ in $\mathrm{A}_{1}\|\psi(\mathrm{x})-\Phi(\mathrm{x})\| \leq$

$$
\begin{aligned}
& \|\psi(x)-\Gamma(x)\|+\left\|\Gamma(x)-\Phi^{n}(I)^{\frac{1}{2}} \Gamma(x) \Phi^{n}(I)^{\frac{1}{2}}\right\|+\left\|\Phi^{s}(x)\right\| \leq \\
& 12, I k^{\frac{1}{2}}+2\left\|\Phi^{n}(I)-I\right\|+2 k(I-2 k)^{-1} \leq \\
& \left(12, I k^{\frac{1}{2}}+6 k(1-2 k)^{-1}\right) \leq 12,1 k^{\frac{1}{2}}+6,02 k
\end{aligned}
$$

and

$$
\|\psi(x)-x\| \leq\|\psi(x)-\Phi(x)\|+\|x-\Pi(x)\| \leq 12,1 k^{\frac{1}{2}}+8,02 k<12,8 k^{\frac{1}{2}}<1
$$

so $\psi$ is injective.

For any $y$ in $B_{1}$ there exists $x$ in $A_{1}$ such that $\|x-y\| \leq k$.

$$
\|\psi(x)-y\| \leq\|\psi(x)-\Phi(x)\|+\|\Phi(x)-y\| \leq 7,02 k+12,1 k^{\frac{1}{2}}<12,8 k^{\frac{1}{2}}<1 .
$$

An argument similar to the one used in the proof of Theorem 3.4 shows then that $\psi$ is an isomorphism of $A$ onto $B$, and the theorem is then a consequence of the following proposition.

I have been told (E.Størmer) that A. Connes has formulated a similar proposition and given a similar proof to it.

\subsection{Proposition.}

Let $\Phi$ be an isomorphism of a von Neumann algebra $A$ with property $M$ onto a von Neumann algebra $B$ acting on the same Hilbertspace $H$. 
If for any $x$ in $A_{1}\|\Phi(x)-x\| \leq k<1$ then there exists a unitary $w$ in $(A \cup B)^{\prime \prime}$ such that

$$
\begin{aligned}
& \Phi(x)=w^{*} x w \quad \text { for all } x \text { in } A \text { and } \\
& \|I-w\| \leq 2^{\frac{1}{2}} k\left(I+\left(I-k^{2}\right)^{\frac{1}{2}}\right)^{-\frac{1}{2}} \text {. }
\end{aligned}
$$

Proof.

Consider the Hilbertspace $\mathrm{H} \oplus \mathrm{H}$ and the von Neumann algebra $\mathrm{C}$ on this space given by

$$
C=\left\{\left(\begin{array}{ll}
a & 0 \\
0 & \Phi(x)
\end{array}\right) \mid a \in A\right\}
$$

Since $C$ has property $M$ there exists an operator $x$ in

$$
\overline{\mathrm{co}}_{\mathrm{C}}\left(\begin{array}{ll}
0 & 1 \\
0 & 0
\end{array}\right) \mathrm{CC}^{\prime}
$$

Take a unitary $u$ in $A$ then

$$
\left(\begin{array}{ll}
u^{*} & 0 \\
0 & \Phi\left(u^{*}\right)
\end{array}\right)\left(\begin{array}{ll}
0 & 1 \\
0 & 0
\end{array}\right)\left(\begin{array}{ll}
u & 0 \\
0 & \Phi(u)
\end{array}\right)=\left(\begin{array}{ll}
0 & u^{*} \Phi(u) \\
0 & 0
\end{array}\right)
$$

and we see that $x$ has the form $x=\left(\begin{array}{ll}0 & y \\ 0 & 0\end{array}\right)$ for some $y$ in $(A \cup B)^{\prime \prime}$ and that $y$ has the property that for any $u$ in $A u^{*} y \Phi(u)=y$ and

$$
\|I-y\| \leq \sup _{u \in A_{u}}\left\|I-u^{*} \Phi(u)\right\| \leq \sup _{x \in A_{I}}\|x-\Phi(x)\| \leq k<1
$$

Let wa be the polardecomposition then standard technique shows that for any $x$ in $A \quad w^{*} x w=\Phi(x)$ and Lemma 2.7 of 6 shows that $w$ is unitary and

$$
\|I-w\| \leq 2^{\frac{1}{2}} k\left(1+\left(1-k^{2}\right)^{\frac{1}{2}}\right)^{-\frac{1}{2}}
$$

4.3 Remark.

Proposition 4.2 needs not hold only for type $M$ algebras, we actually think that proposition 4.2 might be valid in general or perhaps with individual bounds 
on $\mathrm{k}$ for different algebras.

It is easy to check that the only two points where property $M$ is used in the precedent is, the proof of Lemma 3.3 and the proof of proposition 4.2. Actually it is so that we do not need anything more thar proposition 4.2 to prove Lemma 3.3 .

Consider a situation $(\pi(A), p, K)$ as in Lemma 3.3, where $p$ nearly commutes with $\Pi(A)$ then the isomorphism of $\Pi(A)$ given by $\Pi(x) \rightarrow(2 p-I) \Pi(x)(2 p-I)$ is close to the identity isomorphism and the assumption on A shows that there exists a unitary $w$ in $(\pi(A) \cup p)^{\prime \prime}$ such that $w$ is close to $I$ and $w^{*}(2 p-I) \in(\Pi(A) \cup p)^{\prime \prime} n \Pi(A)^{\prime}$, and we are in the situation where the original proof may continue.

Next we want to show that proposition 4.2 is valid when $A$ and $B$ are subalgebras of a finite algebra.

4.4 Proposition.

Let $A$ be a finite von Neumann algebra, $B$ and $C$ sub von Neumann algebras. $\Phi$ and isomorphism of $B$ onto $C$ satisfying $\sup _{x \in B_{1}}\|x-\Phi(x)\| \leq k<1$, then there exists a unitary $u$ in $A$ such that for each $x$ in $B$

$$
u^{*} x u=\Phi(x) \quad \text { and } \quad\|I-u\| \leq 2^{\frac{1}{2}} k\left(I+\left(1-k^{2}\right)^{\frac{1}{2}}\right)^{-\frac{1}{2}}
$$

Proof.

Let $K$ denote the $\sigma-w e a k l y$ closed convex hull of the set of operators $\Phi\left(u^{*}\right) u$ with $u$ unitary in $B$, we will then use the Ryll-Nardzewski's Fixed Point Theorem to prove the existence of $a \quad z$ in $K$ such that

$$
\Phi\left(u^{*}\right) \mathrm{zu}=\mathrm{z} \quad \text { for every unitary } u \text { in } \mathrm{B} \text {. }
$$

The topology which we will consider on A is the ultra-strong topology given by the seminorms $x \rightarrow \varphi\left(x^{*} x\right), \varphi$ a positive normal functional on $A$. 
The weak topology corresponding to this is the o-weak topology, a topology in which $\mathrm{K}$ is compact.

Take $x$ and $y$ in $K$, and suppose $x \neq y$ then there exists a normal finite trace $\tau$ on $A$ such that $\tau\left((x-y)^{*}(x-y)\right) \neq 0$, but then since

$$
\tau\left(\left(\Phi\left(u^{*}\right)(x-y) u\right)^{*}\left(\Phi\left(u^{*}\right)(x-y) u\right)\right)=\tau\left((x-y)^{*}(x-y)\right)
$$

the action $x \rightarrow \Phi\left(u^{*}\right) x u$ of the unitary group in $B$ on $K$ is non contracting and there exists a common fixpoint $z$ in $K,([2])$.

The rest of the proof follows from the proof of proposition 4.2 .

\section{Perturbation results for $\mathrm{C}$ algebras.}

Unfortunately the methods which we have used in the von Neumann algebra case are not strong enough to show unitary equivalence for two close $C^{*}$ algebras, whose weak closures have property $M$, but the methods can for strongly amenable $C^{*}$ algebras $O 2$ and $B$ show that the second dual of $\sigma$ is isomorphic to the second dual of $B$. John Phillips showed in [15] that for two close $c^{*}$ algebras $O r$ and $B$ there exists an orderisomorphism between the set of ideals, an isomorphism which is deternined in such a way that the image of an ideal $\mathcal{Y}$ in $\sigma$ is the only ideal $y$ in $B$ which is close to $y$.

Let us consider the $C^{*}$ algebra $C(H)$ of all compact operatcrs on a Hilbertspace $\mathrm{H}$, is it well known that the second dual of $\mathrm{C}(\mathrm{H})$ is $\mathrm{B}(\mathrm{H})$ and that $\mathrm{C}(\mathrm{H})$ is an idegl of $B(H)$.

In [3] Berglund proves that the only $\mathrm{C}^{*}$ algebras which has the property that they are ideals in their second duals are sub-algebras of $\mathrm{C}(\mathrm{H})$ for some Hilbertspace H. Algebras with this property are called ideal or dual $C^{*}$ algebras.

\subsection{Theorem.}

Let $O L$ and $B$ be $c^{*}$ algebras on a Hilbertspace $H$, if $\|Q C-B\| \leq k<1 / 600$ and $\mathscr{O}$ is ideal then there exists a unitary $u$ in (OGUB)" such that 
$u^{*} B u=\sigma \quad$ and $\quad\|I-u\| \leq 299 k$

Proof.

Since $\leadsto$ is isomorphic to a subalgebre of the algebra of compact operators on some Hilbertspace, the weak closure $\bar{\sigma}$ of $\sigma$ is of type $I$.

If we then use Theorem 4.1 of [6] and the result $u$ in [12] that $\|\overline{C Z}-\bar{B}\| \leq \mathrm{k}$, we may find a unitary $u$ in $(O \mathcal{G} \cup B) "$ such that $\|I-u\| \leq 299 k$ and $u^{*} \bar{B} u=\bar{C}$. The second dual of $O$ has the property that any representation $I I$ of $\sigma$ can be lifted to a normal representation of the second dual onto the weak closure of $\Pi(O)$. If we use this property on the identical representation of $d i$ on $H$, we see that $Q$ is an ideal of $\bar{\sigma}$.

Define $\mathscr{E}=\mathrm{u}^{*} \boldsymbol{B}_{\mathrm{u}}$ then

$$
\|O C-\mathscr{E}\| \leq\|O L-B\|+\|B-\mathscr{C}\| \leq \mathrm{B}+2\|I-u\|<599 / 600<1 .
$$

The work [15] shows that for any representation $I$ of $\bar{\alpha}$,

$$
\|\Pi(\sigma)-\Pi(\mathscr{b})\| \leq\|\sigma-\mathscr{E}\|<1
$$

Consider a representation $\pi$ of $\bar{\sigma}$ with kernel $\mathscr{U}$ then the inequality above shows that $\mathscr{C}$ must be contained in the kernel of $\pi$, hence $\mathscr{C}$ is an ideal $c^{*}$ algebra. By symmetry $O \mathcal{C}=\mathscr{C}$ and the theorem follows.

\subsection{Lemina.}

Let $T$ be locally compact space, let $f$ be a bounded complex function on $T$, and let $k$ be a positive real number less than one.

If to any polynomial $p(f)$ of $f$ and $I$ there is a continuous function $g$, such that $\|p(f)-g\| \leq k\|p(f)\|$ then $f$ is continuous. 
Proof.

We will let $C(T)$ denote the bounded continuous functions on $T$.

If we suppose that $f$ is not continuous, then there will be two different characters $\xi$ and $\eta$ on the $C^{*}$ algebra generated by $C(T)$ and $f$, whose restrictions to $C(T)$ coincide.

Since $f(\xi) \neq f(\eta)$ there exists a polynomial $p$ such that $\|p(f)\| \leq I$; $p(f)(\xi)<-\frac{1+k}{2}$ and $p(f)(\eta)<\frac{1+k}{2}$, but for any $g$ in $C(T)$ with $\|g-p(f)\| \leq k\|p(f)\|$.

$1+k<|p(f)(\xi)-p(f)(n)| \leq|p(f)(\xi)-\xi(\xi)|+|g(n)-p(f)(n)| \leq 2 k<1+k$. $f$ is then in $C(T)$ and the lemma follows.

\subsection{Theorem.}

Let $\sigma$ be a commutative $C^{*}$ algebra on a Hilbertspace $H$ and let $B$ be a

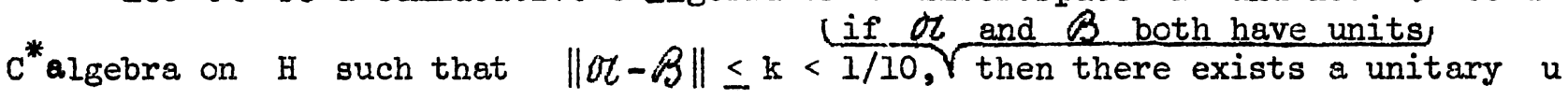
in $(O \in B)^{\prime \prime}$ such that $u^{*} B u=O r$ and

$$
\|I-u\| \leq 2^{5 / 2} k\left(1+\left(1-16 k^{2}\right)^{\frac{1}{2}}\right)^{-\frac{1}{2}}
$$

Proof.

Use Cor. 3.3 in [6] to find a unitary $u$ in $(0 \cup \cup B)^{\prime \prime}$ such that $u^{*} \bar{B} u=\bar{\sigma}$ and

$$
\|I-u\| \leq 2^{5 / 2} k\left(I+\left(1-16 k^{2}\right)^{\frac{1}{2}}\right)^{-\frac{1}{2}}
$$

then

$$
\left\|u^{*} B u-\alpha u\right\| \leq\|q-B\|+2\|I-u\|<I \text {, }
$$

and Lemma 5.2 shows that $u^{*} B u \doteq \mathscr{Z}$. 
In [12] Kadison and Kastler suggest that neighbouring representations are unitarily equivalent, a suggestion which partially was settled in the afirmative in [15]. Here we want to prove a sharper version of the result in [15], a version which with respect to limits on the constant $k$ is best possible for the formulation.

\subsection{Theorem.}

Let $O L$ be a $C^{*}$ algebra and let $\Pi$ and $\rho$ be non degenerated neighbourhing representations of $O Z$ on a Hilbertspace $H$ with

$$
\sup _{x \in \alpha_{1}}\|\Pi(x)-\rho(x)\| \leq k<1
$$

If either $(\pi(\sigma)) "$ has property $M$ or $(\Pi(\sigma) \cup \rho(\sigma)) "$ is finite then $\Pi$ and $\rho$ are equivalent via a unitary $u$ in $(\pi(\sigma) \cup \rho(\sigma)$ " such that

$$
\|I-u\| \leq 2^{\frac{1}{2}} k\left(I+\left(I-k^{2}\right)^{\frac{1}{2}}\right)^{-\frac{1}{2}}
$$

Proof.

Write $\operatorname{Ker}(\pi)$ and $\operatorname{Ker}(\rho)$ for kernels of $\Pi$ and $\rho$ and consider the restriction of $\pi$ to $\operatorname{Ker}(\rho)$, then the assumption on $\pi$ and $\rho$ shows that

$$
\Pi\left(\left(\operatorname{Ker}(\rho)_{1}\right) \leq\{x \in \Pi(\operatorname{Ker}(\rho)) \mid\|x\| \leq k\},\right.
$$

which can only be the case if $\Pi(\operatorname{Ker}(\rho))=0$.

By symmetry we get $\operatorname{Ker}(\pi)=\operatorname{Ker}(\rho)$ and we may define an isomorphism $\Phi^{\prime}$ of $\Pi(\sigma)$ onto $\rho(\sigma)$ by $\Phi^{\prime}(\pi(x))=\rho(x)$.

Our next task is to show that we can extend $\Phi^{\prime}$ to an isomorphism of $\overline{\Pi(\sigma)}$ onto $\overline{\rho(\sigma)}$.

In order to do that we want to consider the space $\mathrm{H} \bigoplus \mathrm{H}$ again and the algebra

$$
B=\left(\begin{array}{ll}
z & 0 \\
0 & \Phi^{\prime}(z)
\end{array}\right), \quad z \in \Pi(\sigma) .
$$


Suppose that for some $z$ in $\overline{\pi(O C)},\left(\begin{array}{ll}z & 0 \\ 0 & 0\end{array}\right)$ belongs to $\bar{B}$, then the Kaplansky density Theorem shows that there exists a net $\left(z_{\alpha}\right)_{\alpha \in D}$ in $\Pi(O C)$ with $\left\|z_{\alpha}\right\| \leq\|z\|$ such that $z_{\alpha}$ converges strongly to $z$ and $\Phi^{\prime}\left(z_{\alpha}\right)$ converges strongly to 0 , but then $z_{\alpha}-\Phi^{\prime}\left(z_{\alpha}\right)$ converges strongly to $z$ and

$\|\mathrm{z}\| \leq \lim \sup \left\|\mathrm{z}_{\alpha}-\Phi^{\prime}\left(\mathrm{z}_{\alpha}\right)\right\| \leq \lim \sup \mathrm{k}\left\|\mathrm{z}_{\alpha}\right\|$, so $\mathrm{z}=0$.

It is now clear that we have got a bijective linear extension $\Phi$ of $\Phi^{\prime}$ from $\overline{\Pi(O)}$ onto $\overline{\rho(\sigma)}$, by defining for $\left(\begin{array}{ll}x & 0 \\ 0 & y\end{array}\right) \in \overline{\mathscr{B}}, \quad \Phi(\mathrm{x})=\mathrm{y}$.

Since $\bar{B}$ is a selfadjointalgebra it also follows that $\Phi$ is a * isomorphism, in fact $\left(\begin{array}{ll}x & 0 \\ 0 & \Phi(x)\end{array}\right)\left(\begin{array}{ll}y & 0 \\ 0 & \Phi(y)\end{array}\right)=\left(\begin{array}{ll}x y & 0 \\ 0 & \Phi(x) \Phi(y)\end{array}\right)$

so $\Phi(x y)=\Phi(x) \Phi(y)$.

Moreover the Kaplansky density theorem shows again that

$$
\sup _{x \in \pi(\sigma)}\|x-\Phi(x)\| \leq k
$$

so we may apply proposition 4.2 or 4.4 to get the desired conclusion. 


\section{References:}

[1] Arveson, W.B., Subalgebras of $C *$ algebras Acta Math., 123 (1969), 141-224.

[2] Asplund, E. and Namolka, I., A geometric proof of RyllNardzewsk1's fixed point theorem. Bull.Amer.Math.Soc., 73 (1967), 443-445.

[3] Berglund, M.C.F., Ideal C*algebras. Duke Math.J., 40 (1973), 241-258.

[4] Bunce, J., Characterizations of amenable and strongly amenable $\mathrm{C}^{*}$ algebras. Pac.J.Math., 43 (1972), 563-572.

[5] Cambern, M., On 1somorph1sms with small bound. Proc.Amer.Math.Soc., 18 (1967), 1062-1066.

[6] Christensen, E., Perturbations of type I von Neumann algebras, To appear in J.London Math. Soc.

[7] Dixmier, J., Les algebres d'opérateurs dans l'espace Hilbertien, deuxieme edition, Gauthier-Vilars, Paris, 1969.

[8] Dixmier, J., Les $C^{*}$-algebres et leurs representations, Gauthier-Vilars, Paris, 1964.

[9] Kadison, R.V., Isometries of operator algebras. Ann. of Math., 54 (1951), 325-338.

[10] Kadison, R.V., A generalised. Schwarz Inequality and algebraic invariants for operator algebras, Ann.of Math.56(1952), 494-503.

[1I] Kadison, R.V., Derivations of operator algebras. Ann. of Math., 83 (1966), 280-293.

[12] Kadison, R.V. and Kastler, D., Perturbations of von Neumann algebras I stability of type. Amer.J.Math., 94 (1972), 38-54.

[13] Lieb, E.H. and Ruskai, M.B., Some operator inequalities of Schwarz type. To appear. 
[14] Phililps, J., Perturbations of type I von Neumann algebras, to appear in Pac. J. Math.

[15] Phillips, J., Perturbations of $C^{*}$-algebras, to appear in Indiana Univ. Math. J.

[16] Phillips, J., Neighbouring representations of certain $C^{*}$ algebras. Mathematical Preprint No. 1973 - 35, Queens University, Kingston.

[17] Saka1,S., C $C^{*}$-algebras and $W^{*}$-algebras. Springer-Verlag, 1971.

[18] Schwartz,J., Two finite, non-hyperfinite, non-isomorphic factors, Comm.Pure Appl. Math., 16 (1963), 19-26.

[19] Stinespring, W.F., Positive functions on $C^{*}$-algebras, Proc.Amer.Math.Soc., 6 (1955), 211-216.

[20] Takesaki, M., On the conjugate space of operator algebra, Tôhoku Math. J., 10 (1958), 194-203.

[21] Tomiyama, J., On the projection of norm one in $\mathrm{W}^{*}$-algebras, III, Tôhoku Math. J., 11 (1959), 125-129.

[22] Tomiyama, J., On the tensorproduct of von Neuman algebras, Pac.J.Math., 30 (1969), 263-270.

[23] Tomiyama, J., Tensorproducts and projections of norm one in von Neumann algebras, Seminar, University of Copenhagen, 1970.

[24] Tomiyama, J., The extension property and tensorproducts of $C^{*}$-algebras, Nordic summer school in math., Copenhagen, 1973. 